\title{
Como padrões climáticos afetam a intensidade de seleção sexual sobre atributos sexualmente dimórficos?
}

\author{
$\underline{\text { Douglas Fernandes }}$; Gilberto M. M. Santos ${ }^{2}$ \\ $1-$ \\ Bolsista PIBIC/CNPq, Graduando em Ciências Biológicas, Universidade Estadual de Feira de \\ Santana, e-mail: \\ macieluefs@gmail.com \\ 2- Orientador, Gilberto M. M. Santos, Universidade Estadual de Feira de Santana. Departamento \\ Biologia.
}

PALAVRA-CHAVE: Intensidade de seleção sexual, dimorfismo sexual, interações agonistas; macroecologia da seleção sexual.

\section{Introdução}

A seleção sexual é subdividida em dois processos: seleção intra-sexual e seleção inter-sexual. A seleção intra-sexual ocorre quando há competição direta entre indivíduos de mesmo sexo e mesma espécie pelo acesso a indivíduos do sexo oposto (e.g. Junior \& Peixoto 2013; Painting \& Holwell 2014). Já a seleção inter-sexual ocorre quando atributos de um indivíduo conferem vantagem por aumentar a chance de serem escolhidos por indivíduos do sexo oposto (e.g. Cade \& Cade 1992; Watson et al. 1998).

Diferentes características sexualmente dimórficas dos machos podem ser favorecidas tanto por competição intra quanto inter-sexual (Clutton-Brock, 2014). Porém investir nessas características gera custos para os indivíduos (Allen \& Levinton, 2007). Portanto, estes individuos só produzem estas carateristicas quando eles têm alta vantagem reprodutiva (alta intencidade de seleçao sexual) (Andersson 1994).

Há vários fatores que podem afetar a intensidade de seleção sexual sobre estesatributos sexualmente dimorficos (Emlen \& Oring, 1977). Porem alguns fatores com a estabilidade climática de onde as espécies ocorrem pode ser desproporcionalmente mais importante por afetar simultaneamente várias características do sistema de acasalamento. Quanto maior a estabilidade, maior deve ser a janela reprodutiva adequada e consequentemente, maior a assincronia de receptividade sexual de fêmeas. Consequentemente, em climas estáveis os machos devem ter oportunidade para monopolizar as fêmeas, o que aumenta a pressão de seleção sobre estruturas sexualmente dimórficas que assegurem a vitória em competições por fêmeas. Portanto, nesse trabalho, desenvolvemos uma meta-análise com o objetivo de avaliar se existe um padrão previsível de investimento em atributos sexualmente dimórficos em machos que brigam pelo acesso a fêmeas propomos a hipótese de que locais com maior estabilidade climática favorecem o maior investimento em estruturas sexualmente dimórficas usadas entre machos em competições por acesso a fêmeas. Uma das formas de se medir a intensidade de seleção sexual é pelo uso do gradiente de seleção sexual que é obtido por meio de regressões lineharém entre uma medida de sucesso reprodutivo e o atributo (Lande \& Arnold, 1983). Sendo assim, se nossa hipótese for verdadeira, esperamos encontrar maior gradiente de seleção sexual em atributos sexualmente dimórficos com o aumento da estabilidade climática. 


\section{Métodos}

Para coleta de dados, fizemos buscas por artigos nas bases do Google Acadêmicos (https://scholar.google.com.br/), ISI Web of Science, e também em nossa base de artigos pessoais. Para as buscas usamos as palavras-chave: sexual selection e selection intensity. coletamos informações sobre 1) a inclinação das retas de regressões lineharém entre o tamanho do atributo sexualmente dimórfico usado em competições por acesso a fêmeas e sucesso reprodutivo dos indivíduos assim com o erro padrão associado a essa inclinação, 2) o local onde o estudo foi realizado, 3) o número de amostras usadas nas análises, 4) a espécie estudada.

Para determinar a estabilidade climática do local onde cada espécie foi estudada usamos a classificação climática de Köppen (http://koeppen-geiger.vu-wien.ac.at/). . De acordo com essa classificação, os climas Af (equatorial) e Am (equatorial) devem ser aqueles com maior estabilidade climática. climas classificados como Aw (savanas tropicais), C (temperados) e D (frios) são considerados intermediários. Já ocais classificados como BSk (árido), BWk (árido), BSh (árido), BWh (árido) e E (polar) são considerados instáveis.

\section{Resultados}

Nossas buscas resultaram em 1.361 artigos. Desse total, 20 (representando 20 espécies distintas) apresentaram dados adequados para análise. Muitos trabalhos mediram o valor do gradiente para mais de uma característica e em populações distintas da mesma espécie (e.g. Puniamoorthy et al 2011; Reichard et al 2008; Wacker et al 2015). Por isso, obtivemos 99 medidas de gradiente. Encontramos 3 espécies e 13 medidas de gradiente em espécies sob climas intermediário e 17 espécies e 99 medidas de gradiente em espécies sob climas instáveis. Não encontramos valores de gradiente para espécies sob climas estáveis. Como há uma diferença bem definida entre os climas intermediários e instáveis, seguimos com as análises mantendo nossas previsões originais.

O teste referente ao valor do gradiente em relação às condições climáticas não indicou relação entre o clima e os atributos sexualmente dimórficos $(\mathrm{QM}=0,0056, \mathrm{df}=1, \mathrm{p}=$ 0,94 fig 1). 


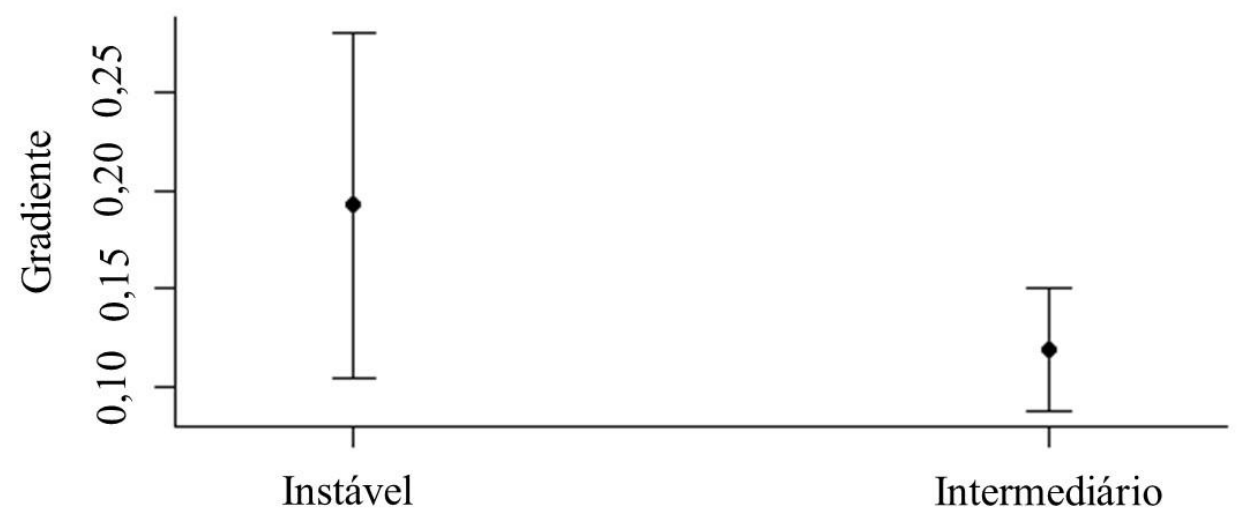

Estabilidade Climática

Figura 1 Relação entre a estabilidade climática e o valor do gradiente de seleção sexual de atributos sexualmente dimórficos usados pelos machos nas competições por acesso as fêmeas. No total usamos 20 espécies de animais em 112 amostras de gradiente. Encontramos 3 espécies (13 amostras de gradiente) em climas instáveis e 17 espécies (99 amostras) em climas intermediários.

\section{Discussão}

O valor do gradiente não variou em função da estabilidade climática. Isso sugere que o aumento da estabilidade climática não favorece o aumento da intensidade de seleção sexual sob atributos sexualmente dimórficos dos machos. Logo, Indivíduos com alto investimento em estruturas dimórficas, provavelmente, não são favorecidos pela alta sincronia de emergência das fêmeas.

\section{Conclusão}

Aestabilidade climática não parece ser um fator hierarquicamente mais importante para explicar o aumento do investimento em atributos sexualmente dimórficos usados em competições entre os machos por acesso as fêmeas. Acreditamos que isso acontece já que a estabilidade afeta a sincronia de emergência das fêmeas, mas a sincronia de emergência das fêmeas não parece afetar a capacidade dos machos monopoliza-las. 


\section{Referência}

Allen, B. J. \& Levinton, J. S. 2007. Costs of bearing a sexually selected ornamental weapon in a fiddler crab. Functional Ecology, 21, 154-161.

Andersson, M. B. 1994. Sexual selection. Princeton: Princeton University Press.

Cade, W. H. \& Cade, E. S. 1992. Male mating success, calling and searching behaviour at high and low densities in the field cricket, Gryllus integer. Animal Behaviour, 43, 49-56.

Clutton-Brock, T. 2014. Sexual selection in males and females. Science, 318, 1882-1885.

Emlen, S. \& Oring, L. 1977. Ecology, sexual selection, and the evolution of mating systems. Science, 85, 663-669.

Junior, R. S. L. \& Peixoto, P. E. C. 2013. Males of the dragonfly Diastatops obscura fight according to predictions from game theory models. Animal Behaviour, 85, 663669.

Painting, C. J., \& Holwell, G. I., 2014. Exaggerated rostra as weapons and the competitive assessment strategy of male giraffe weevils. Behavioral Ecology, 25, 1223-1232.

Puniamoorthy N. Schafer M. A. Blanckenhorn W.U. 2012. sexual selection accounts for the geographic reversal of sexual size dimorphism in the dung fly, Sepsis punctum (Diptera: Sepsidae). Evolution, 66, 2117-2126.

Reichard, M., Ondračková, M., Bryjová, A., Smith, C., \& Bryja, J. 2009. Breeding resource distribution affects selection gradients on male phenotypic traits: experimental study on lifetime reproductive success in the bitterling fish (Rhodeus amarus). Evolution 63:377-390.

Wacker, S., Mobley, K., Forsgren, E., Myhre, L. C., Jong, K., \& Amundsen, T. 2013. Operational sex ratio but not density affects sexual selection in a fish. Evolution 67, 1937-1949.

Watson, P. J. Arnqvist, G. \& Stallmann, R. R. 1998. Sexual conflict and the energetic costs of mating and mate choice in water dtriders. American Naturalist, 151, 4658. 